\title{
EVALUACIÓN DEL ACEITE ESENCIAL DE ORÉGANO (Origanum vulgare) Y EXTRACTO DESHIDRATADO DE JENGIBRE (Zingiber officinale) COMO POTENCIALES PROMOTORES DE CRECIMIENTO EN POLLOS DE ENGORDE
}

\author{
Oregano Essential Oil (Origanum Vulgare) and Ginger Dried Extract \\ (Zingiber officinale) as Potential Growth Promoters in Broilers
}
Carlos Shiva $^{1,5}$, Samuel Bernal ${ }^{1}$, Michel Sauvain ${ }^{3}$, Justina Caldas ${ }^{4}$, Juan Kalinowski ${ }^{4}$, Néstor Falcón ${ }^{1}$, Rosario Rojas ${ }^{2}$

\section{RESUMEN}

Se evaluó el efecto de la adición en la dieta del aceite esencial de orégano (Origanum vulgare) y de jengibre deshidratado (Zingiber officinale) en la ganancia de peso, consumo de alimento e índice de conversión alimenticia en 624 pollos machos Cobb 500 distribuidos aleatoriamente en cuatro tratamientos y cuatro repeticiones de 39 broilers cada una. Se obtuvieron muestras de intestino e hígado de cuatro aves por tratamiento el día 14 y dos por tratamiento el día 42 del ensayo para determinar posibles alteraciones histológicas por intoxicación y para determinar la histomorfometría de yeyuno. Los tratamientos fueron: APC, Bacitracina disalicilato metileno ( $1 \mathrm{~kg} / \mathrm{TM}$ de alimento) y sulfato de colistina 8\% (0.25 kg/TM); SPC, sin promotor de crecimiento; AEO, aceite esencial de orégano (1 kg/TM); y JD, jengibre deshidratado (10 kg/TM). No hubo diferencias estadísticas en peso, consumo de alimento ni conversión alimenticia entre grupos experimentales. Las vellosidades del grupo AEO presentaron una ligera aunque no significativa mayor longitud de vellosidades intestinales. Las aves de todos los grupos presentaron alteraciones histológicas en yeyuno e hígado aunque con diferente intensidad. Se concluye que el uso de AEO y JD como promotores de crecimiento no difieren de los otros tratamientos, probablemente por un bajo reto sanitario.

Palabras clave: broiler, promotor de crecimiento, aceite esencial de orégano, jengibre deshidratado

\footnotetext{
${ }^{1}$ Facultad de Veterinaria y Zootecnia, ${ }^{2}$ Facultad de Ciencias y Filosofía, Universidad Peruana Cayetano Heredia, Lima, Perú

${ }^{3}$ MR 152 «PHARMA-DEV» Institut de Recherche pour le Développement, Universidad de Toulouse, Francia

${ }^{4}$ San Fernando S.A., Lima, Perú

${ }^{5}$ E-mail: carlos.shiva@upch.pe
} 
The effect of adding oregano (Origanum vulgare) essential oil and dry ginger (Zingiber officinale) in the diet on body weight gain, feed intake and feed conversion rate was evaluated in 624 male Cobb 500 broiler chicks randomly assigned into four treatments with four replicates of 39 chicks each. Intestine and liver samples from four broilers per treatment were collected at day 14 and two at day 42 after treatment to determine possible histological toxic alterations and to determine the intestinal histomorphometry of jejunum. The treatments were: APC, Bacitracin methylene disalicylate (1 kg/MT of feed) and colistine sulfate $8 \%(0.25 \mathrm{~kg} / \mathrm{MT})$; SPC, without growth promoter; AEO, oregano essential oil ( $1 \mathrm{~kg} / \mathrm{MT})$; and JD, dryy ginger (10 kg/MT). There was no statistical difference between groups in body weight gain, feed intake and feed conversion rate. Group AEO showed slightly larger villi but without statistical differences with other groups. All birds showed alterations in jejunum and liver. It is concluded that the use of AEO and JD as growth promoter did not improved productive performance probably due to the low health challenge.

Key words: broiler, growth promoter, oregano essential oil, dry ginger

\section{INTRODUCCIÓN}

Ante los indicios de la generación de resistencia antibacteriana producida por el uso de antibióticos promotores de crecimiento (APC) en animales de granja y su impacto en la salud pública, la OMS sugirió su prohibición y retiro del mercado a nivel mundial (WHO, 2001). En respuesta, en el 2003, la Unión Europea promulgó el Reglamento (CE) N. ${ }^{\circ} 1831 / 2003$ sobre los aditivos en la alimentación animal, estableciendo que a partir del 1 de enero de 2006 los antibióticos, coccidiostatos e histomoniatos no pueden ser usados en alimentos para animales (FAO/ OMS, 2005).

Como consecuencia de este hecho, se ha intensificado la búsqueda de alternativas al uso de APC (Torres y Zarazaga, 2002). Dentro de estas posibilidades, se enmarca la gran aceptación de productos naturales, utilizados generalmente como antioxidantes y saborizantes, como el orégano (Origanum vulgare) y el jengibre (Zingiber officinale), con resultados de investigación prometedores en la industria avícola y porcina por su acción antimicrobiana (Windisch et al., 2008).
En el orégano, la actividad antimicrobiana es atribuida, principalmente, a los componentes carvacrol y timol. En el Perú, se ha logrado extraer sus aceites esenciales de hojas y flores secas, obteniendo un $9 \%$ de carvacrol, $12.2 \%$ de terpineol y $6.7 \%$ de pcimeno; sin embargo, su composición es variable, y a veces incluye timol (Albado et al., 2001; Arcila et al., 2004). En ensayos en pollos broiler suplementados con APC como bacitracina y avilamicina y con extracto de $O$. vulgare no se encontraron diferencias significativas en conversión alimenticia o morfometría intestinal (Hernández et al., 2004; Ortiz, 2004; Barreto et al., 2008). Adicionalmente, en un estudio infectando pollos broiler con Eimeria tenella y con adición de aceite de orégano (0.5-1.0 g/ $\mathrm{kg}$ ) en la dieta se obtuvo resultados anticoccidiales similares a otros productos comerciales (Silva et al., 2009).

Se dispone de trabajos in vitro donde se demuestra la propiedades antimicrobianas de Z. officinale, atribuidos a los componentes 6-gingerol y zingerona, pero son escasos los ensayos in vivo. Se ha demostrado in vitro que extractos de jengibre bloquean la unión entre la enterotoxina termolábil de la 
Escherichia coli al receptor de la superficie celular $\mathrm{G}_{\mathrm{M} 1}$ por medio de zingerona (vanillil acetona) (Chen et al., 2007).

Pocos estudios han evaluado el uso del jengibre deshidratado en la producción animal pero hay indicios de que su uso es factible, especialmente en la producción avícola. En un estudio en pollos broilers con diferentes tamaños de partículas de jengibre deshidratado ( $5 \mathrm{~g} / \mathrm{kg}$ de alimento) se obtuvo mayor peso de carcasa con menor peso de grasa abdominal (Zhang et al., 2009); en tanto que con la adición de jengibre deshidratado no se encontró diferencias en peso y conversión alimenticia, pero se observaron alteraciones enterohepáticas (Al-Homidan, 2005). En contraste, otro estudio con jengibre al $0.2 \%$ y una combinación de $0.2 \%$ de jengibre y $0.2 \%$ de pimienta se obtuvo mayores pesos al de control sin plantas deshidratadas (Moorthy et al., 2009).

El objetivo del presente estudio fue evaluar el efecto del aceite esencial de orégano (Origanum vulgare) y del jengibre deshidratado (Zingiber officinale) como reemplazo a antibióticos promotores de crecimiento en dietas para pollos de engorde.

\section{Materiales y MÉTodos}

\section{Ubicación del Estudio}

El estudio se realizó en la granja experimental Valientes, ubicada en el distrito de Chorrillos, de propiedad de la empresa San Fernando S.A. La formulación de las raciones se realizó en la Planta de Alimentos de San Fernando, Lurín, y el procesamiento de las muestras clínicas en los laboratorios de la Universidad Peruana Cayetano Heredia (UPCH), San Martín de Porres, Lima.

Tanto las condiciones de bioseguridad como de manejo de la granja experimental fueron óptimas y acordes con los estándares internacionales de la industria avícola. Se rea- liza un estricto control de plagas y roedores, así como control de entrada de personal y la utilización de vestimenta exclusiva de la granja experimental. La cama no se reutiliza. Asimismo, se registró diariamente la condición de humedad de la cama y la temperatura ambiental en siete puntos del galpón.

\section{Grupos Experimentales}

Se utilizaron 624 pollos de carne machos de la línea Cobb 500, con un peso promedio de 50 g en la recepción al primer día de edad, distribuidos en cuatro dietas con cuatro repeticiones cada una. Cada repetición estuvo conformada por 39 pollos en un área de crianza de $4 \mathrm{~m}^{2}$ y con una densidad de 9.75 pollos $/ \mathrm{m}^{2}$. La duración del ensayo fue de 42 días. Todo animal muerto durante la primera semana fue restituido por otro del mismo peso.

Las dietas cubrían los requerimientos nutricionales de los pollos en todas las fases de su desarrollo. El alimento fue administrado en polvo y el agua se suministró ad libitum. Las dietas, según el tipo de suplemento, fueron las siguientes:

- Dieta APC: Grupo control, con antibiótico promotor de crecimiento (bacitracina metilen disalicilato [BMD $1 \mathrm{~kg} / \mathrm{TM}$ de alimento] y sulfato de colistina [ $8 \% 0.25$ $\mathrm{kg} / \mathrm{TM}$ de alimento]) y sin extracto de plantas.

- Dieta AEO: Con aceite esencial de orégano (1 kg/TM de alimento).

- Dieta JD: Con extracto de jengibre deshidratado (10 kg/TM de alimento).

- Dieta SPC: Sin antibiótico promotor de crecimiento ni extracto de plantas.

Los aditivos no nutricionales fueron retirados de las dietas a partir del día 40, respetando el periodo de retiro de tres días antes del beneficio. El aceite esencial de orégano y el extracto deshidratado de jengibre fueron procesados en la Unidad de Investigación en Productos Naturales de la UPCH. 


\section{Variables en Estudio}

Peso corporal y ganancia de peso. El pesado se realizó el día de recepción y los días 7, 14, 21, 28, 35 y 42. La ganancia de peso semanal se obtuvo por diferencia entre los pesos semanales, y la ganancia de peso acumulada fue la diferencia entre el peso final y peso inicial. El pesaje se hizo en forma grupal (dos grupos por repetición) en jabas. Sólo en la sexta semana se realizaron pesados de los animales de manera individual. El peso se registró con balanzas digitales con precisión de $\pm 0.1 \mathrm{~g}$.

Consumo de alimento. Semanalmente se pesó el alimento sobrante en los comederos de cada corral. Al peso del alimento proporcionado durante la semana se le restó el peso de alimento sobrante y el resultado se dividió entre el número de aves para obtener el consumo de alimento semanal y acumulado por ave.

Conversión alimenticia. La conversión alimenticia (CA) por semana y de la campaña se calculó con las siguientes fórmulas:

- $\quad$ CA semanal = Alimento consumido semanal / Ganancia de peso semanal

- $\quad$ CA acumulada = Alimento consumido por campaña / Ganancia de peso final

Mortalidad. Se realizó la necropsia a todo animal que murió durante el estudio para determinar las causas de mortalidad. Además, se hizo un análisis macroscópico de la integridad intestinal a un ave sacrificada por cada repetición mediante selección aleatoria en la segunda y sexta semana de edad.

\section{Evaluación Histológica}

El examen histológico se realizó en cuatro aves por tratamiento en la segunda semana y en dos aves por tratamiento en la sexta semana de la campaña. Las aves fueron seleccionadas en forma aleatoria. Se tomaron muestras de 3-4 cm de asa intestinal (yeyuno) y $1 \mathrm{~cm}^{2}$ de hígado, y se conservaron en formol al $10 \%$. Se emplearon procedimientos histológicos de rutina y tinción hematoxilinaeosina (H\&E).

Se buscó indicios de toxicidad en los cortes histológicos. Se evaluaron 40 vellosidades por tratamiento en la segunda semana y 30 vellosidades por tratamiento en la sexta semana, determinándose como vellosidades con alteración aquellas que presentaban hiperplasia de enterocitos, hiperplasia de células caliciformes e hiperplasia de células de las criptas de Lieberkuhn con diferente intensidad. La frecuencia de vellosidades intestinales con alteraciones fueron cuantificadas como normal ( 0 a 2), escasa ( 3 a 4), moderada (5 a 7) y severa (8 a 10). En hígado se determinó alteraciones como tumefacción turbia, degeneración hidrópica; además de congestión, infiltración linfocitaria y edema intersticial. Se evaluó la intensidad de cada alteración según su presencia y predominio en todo el campo de la muestra histológica.

Además, se realizó el análisis histomorfométrico de las vellosidades intestinales, determinándose la longitud y ancho de las vellosidades, y la profundidad de las criptas de Lieberkuhn de 10 vellosidades por cada muestra. Para la descripción de las láminas se empleó un microscopio triocular de campo claro (DM LS2, Leica), adaptado con una cámara de microfotografía de 2 megapixeles de resolución (Motic 2000).

\section{Modelo Estadístico}

El modelo estadístico fue un diseño completamente al azar. Para determinar diferencias significativas en los parámetros productivos (ganancia de peso, consumo de alimento y conversión alimenticia), así como de la histomorfometría (longitud y ancho de vellosidades, y profundidad de criptas de Lieberkuhn) se utilizó la prueba de análisis de varianza (ANOVA). La evaluación de grados de alteraciones histológicas en intestino delgado e hígado se hizo con la prueba de Kruskal-Wallis. Los datos se analizaron mediante el programa SPSS 15.0. 


\section{Resultados}

La ganancia de peso semanal fue similar entre grupos (Fig. 1), con excepción del día 14, donde el grupo con la dieta AEO presentó la menor ganancia de peso $(p<0.05)$. No se encontró diferencia estadística en el peso a los 42 días entre grupos ni en el consumo acumulado de alimento durante la campaña (Cuadro 1).

La Fig. 2 muestra que la conversión alimenticia semanal de los pollos broiler durante las seis semanas del ensayo fue similar entre tratamientos. Tampoco se encontró diferencias estadísticas en la conversión alimenticia acumulada al final del ensayo (Cuadro 1).

La mortalidad y descarte de aves en la primera semana fue debido a animales en condiciones de retraso de crecimiento, así como impacción del tracto digestivo, consumo de la cama y retención de yema. Al final de la campaña no se observó diferencias significativas en la mortalidad entre tratamientos (Cuadro 1).

Las aves mostraron alteraciones en el intestino a los 14 días de edad: hiperplasia de las células caliciformes, hiperplasia de los enterocitos e hiperplasia a nivel de las criptas de Lieberkuhn, aunque con diferente grados de severidad. En el grupo APC (control) se observó hiperplasia a los tres niveles con tendencia a ser moderado, en tanto que en los grupos SPC y JD se observó hiperplasia a nivel de enterocitos y criptas de Lieberkuhn, con tendencia a ser moderada. En contraste, el grupo AEO presentó una escasa hiperplasia.

En el día 42, los grupos APC y SPC mantuvieron un grado moderado de hiperplasia de enterocitos, células caliciformes y a nivel de las criptas de Lieberkuhn (Fig. 3), en tanto que en el grupo JD había una moderada hiperplasia de enterocitos y escasa hiperplasia en cripta de Lieberkuhn. En el grupo AOE solo hubo una escasa hiperplasia a nivel de las criptas de Lieberkuhn.

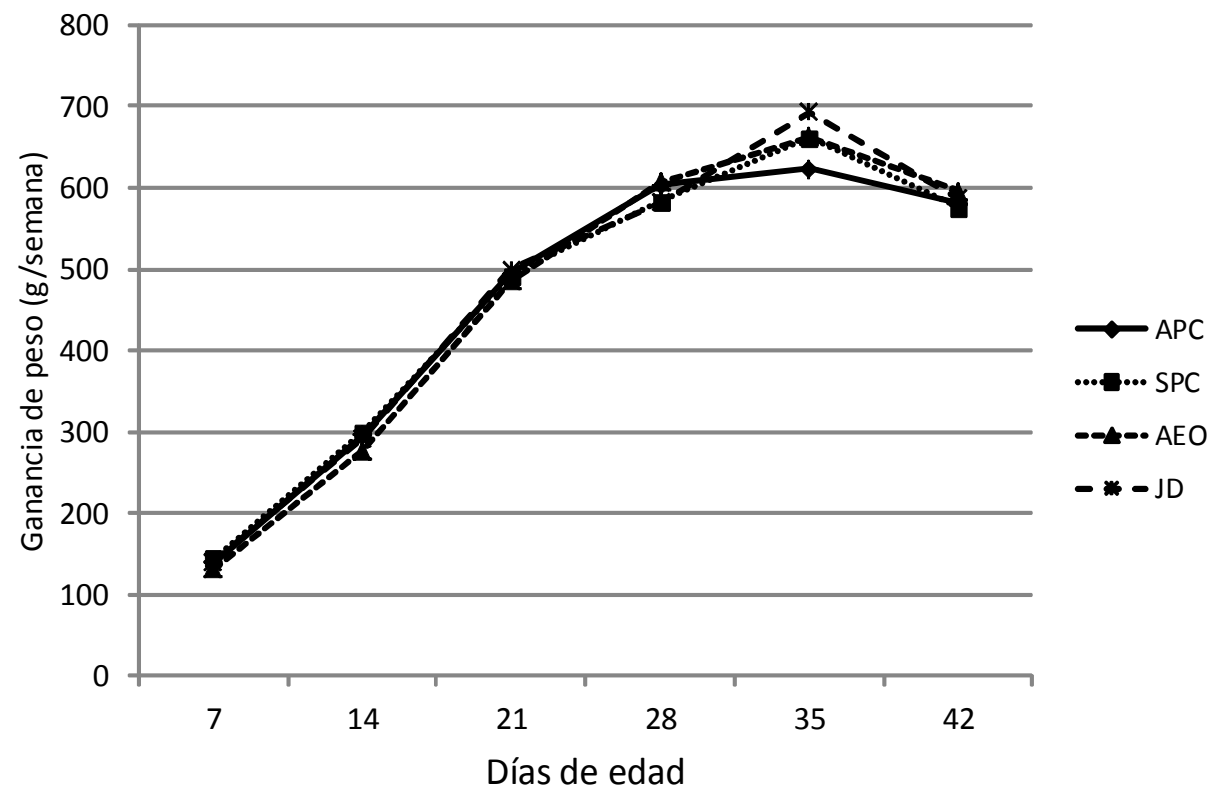

Figura 1. Ganancia de peso semanal (gramos) de pollos broiler suplementados con plantas medicinales. APC: Antibiótico promotor de crecimiento; SPC: Sin promotor de crecimiento; AEO: Aceite esencial de orégano; JD: Jengibre deshidratado 


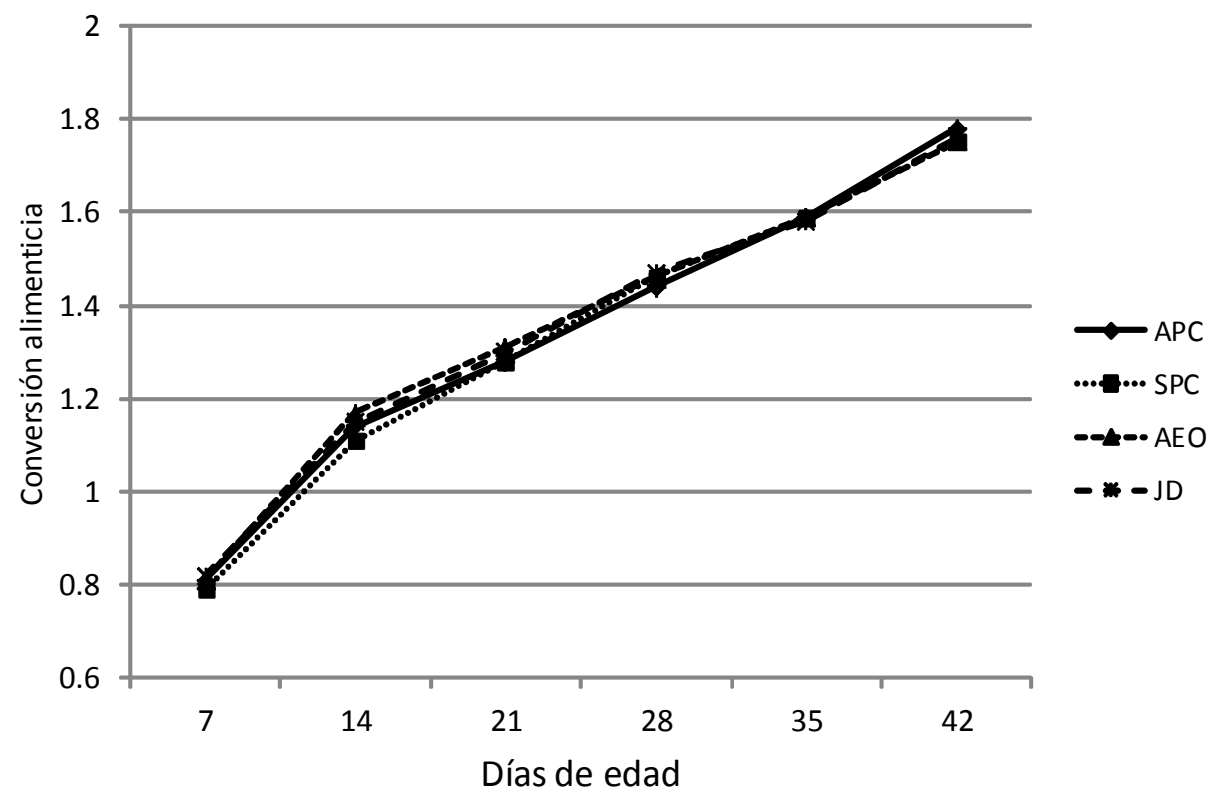

Figura 2. Conversión alimenticia acumulada de pollos broiler suplementados con plantas medicinales. APC: Antibiótico promotor de crecimiento; SPC: Sin promotor de crecimiento; AEO: Aceite esencial de orégano; JD: Jengibre deshidratado

Cuadro 1. Parámetros productivos de pollos broiler a los 42 días de edad que fueron suplementados con extractos de plantas medicinales ${ }^{1}$

\begin{tabular}{lcccc}
\hline \multirow{2}{*}{ Parámetros productivos } & \multicolumn{4}{c}{ Tratamientos $^{2}$} \\
\cline { 2 - 5 } & APC & SPC & AEO & JD \\
\hline Peso corporal (g) & 2788 & 2827 & 2811 & 2847 \\
Consumo de alimento (g) & 4961 & 4920 & 4928 & 5012 \\
Conversión alimenticia & 1.78 & 1.75 & 1.75 & 1.76 \\
Mortalidad (\%) & 1.28 & 1.92 & 0.64 & 1.92 \\
\hline
\end{tabular}

1 El suplemento se retiró de la dieta a los 40 días de edad

2 APC: Antibiótico promotor de crecimiento; SPC: Sin promotor de crecimiento; AEO: Aceite esencial de orégano; JD: Jengibre deshidratado. No hubo diferencia estadística entre grupos

En el caso del hígado, en el día 14 se observó degeneración hidrópica severa en todos los grupos, y además, una moderada tumefacción turbia en el grupo JD (Fig. 4). Estas alteraciones involucionan hasta el día 42, donde sólo se observa una leve congestión y moderada tumefacción turbia en el gru- po APC, una moderada tumefacción turbia en el grupo JD y escasas alteraciones en el grupo AEO.

En el Cuadro 2 se presentan las medidas histomorfométricas de las vellosidades intestinales, sin haber encontrado diferencias estadísticas entre grupos. 
Cuadro 2. Histomorfometría de las vellosidades intestinales (media \pm desviación estándar)

\begin{tabular}{|c|c|c|c|c|}
\hline \multirow{2}{*}{ Medidas $(\mu \mathrm{m})$} & \multicolumn{4}{|c|}{ Tratamiento $^{1}$} \\
\hline & APC & SPC & AEO & JD \\
\hline \multicolumn{5}{|c|}{ A) 14 días de edad $(n=40)$} \\
\hline $\begin{array}{l}\text { Longitud }^{2} \\
\text { Ancho }{ }^{2} \\
\text { Profundidad }{ }^{3}\end{array}$ & $\begin{array}{c}878 \pm 122 \\
86 \pm 21 \\
60 \pm 18\end{array}$ & $\begin{array}{c}774 \pm 110 \\
59 \pm 11 \\
63 \pm 20\end{array}$ & $\begin{array}{c}888 \pm 117 \\
59 \pm 10 \\
60 \pm 18\end{array}$ & $\begin{array}{c}816 \pm 146 \\
72 \pm 21 \\
47 \pm 15\end{array}$ \\
\hline \multicolumn{5}{|c|}{ B) 42 días de edad $(n=30)$} \\
\hline $\begin{array}{l}\text { Longitud }^{2} \\
\text { Ancho }^{2} \\
\text { Profundidad }^{3}\end{array}$ & $\begin{array}{c}834 \pm 100 \\
87 \pm 30 \\
80 \pm 27\end{array}$ & $\begin{array}{c}780 \pm 156 \\
95 \pm 24 \\
59 \pm 18\end{array}$ & $\begin{array}{c}965 \pm 124 \\
87 \pm 20 \\
49 \pm 13\end{array}$ & $\begin{array}{c}681 \pm 175 \\
124 \pm 33 \\
63 \pm 26\end{array}$ \\
\hline
\end{tabular}

\section{Discusión}

El presente estudio demuestra que no existe diferencia estadística entre los grupos suplementados durante 40 días con aceite esencial de orégano $(1 \mathrm{~kg} / \mathrm{TM})$ y el jengibre deshidratado (10 kg/TM) frente a una ración con bacitracina metilen disalicilato (BMD 1 $\mathrm{kg} / \mathrm{TM})$ y sulfato de colistina $8 \%(0.25 \mathrm{~kg} /$ TM ) en pollos Cobb 500 bajo condiciones experimentales optimizadas de control ambiental y sanitario.

El efecto como promotor de crecimiento de los extractos ensayados está relacionado con sus propiedades antibacterianas; en el caso de $O$. vulgare es debido a sus componentes fenólicos carvacrol y timol (Dorman y Deans, 2000; Albado et al., 2001; Morais et al., 2007), que incrementan la permeabilidad de la membrana bacteriana permitiendo que se disipen las gradientes de $\mathrm{pH}$ internos y la salida de iones inorgánicos (Dorman y Deans, 2000; Lambert et al., 2001). En el caso de Z. officinale, la actividad antimicrobiana es producida por el gingerol y el zingerona (Akoachere et al., 2002; Indu et al., 2006; Stoyanova et al., 2006), efecto pro- ducido por la competencia de receptores con enterotoxinas termolábiles, como los de $E$. coli, al unirse al receptor de la superficie celular $\mathrm{G}_{\mathrm{M} 1}$ de las vellosidades intestinales (Chen et al., 2007).

Hashemi y Davoodi (2010) mencionan que el efecto antibacteriano y de promotor de crecimiento están estrechamente relacionados ya que afectan benéficamente el ecosistema microbiano intestinal al controlar las bacterias patógenas y sus toxinas y, en consecuencia, mejorando la digestibilidad de los nutrientes. Este efecto no se vio reflejado en el presente estudio, pues no hubo diferencias significativas entre los grupos tratados y el grupo que no recibió el promotor de crecimiento ni los extractos de plantas. La falta de respuesta puede atribuirse al adecuado manejo ambiental y sanitario en la granja experimental que evitó posibles efectos negativos en el grupo SPC.

En un ensayo que utilizó $0.1 \%$ de extracto de orégano se obtuvo una conversión alimenticia y morfometría hepática similar a la obtenida usando avilamicina, antibiótico promotor de crecimiento (Barreto et al., 2008). Similares, resultados obtuvo Fukayama 


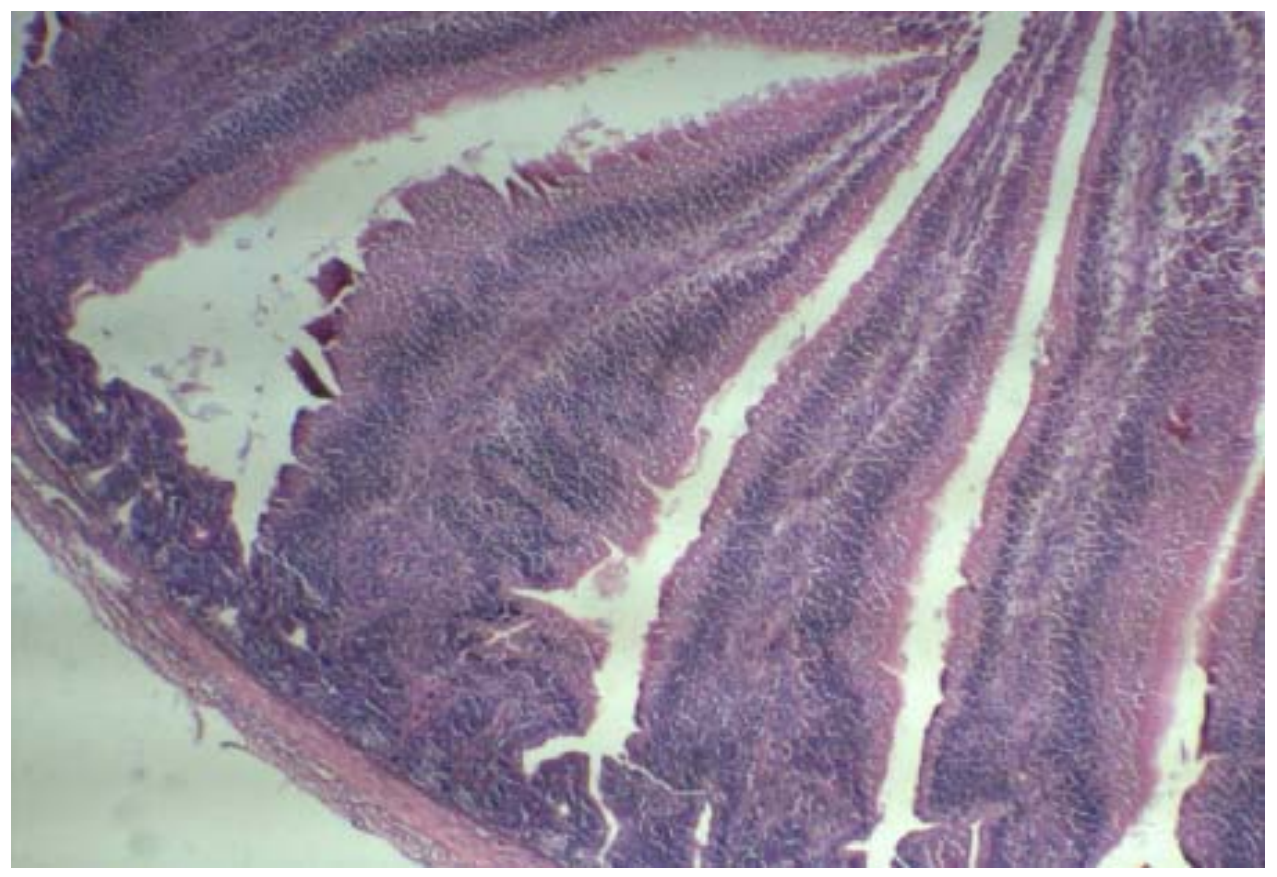

Figura 3. Histología de yeyuno de pollo de 14 días, del grupo control (con antibiótico promotor de crecimiento). Presenta hiperplasia de células caliciformes e hiperplasia de enterocitos en vellosidades (x100)

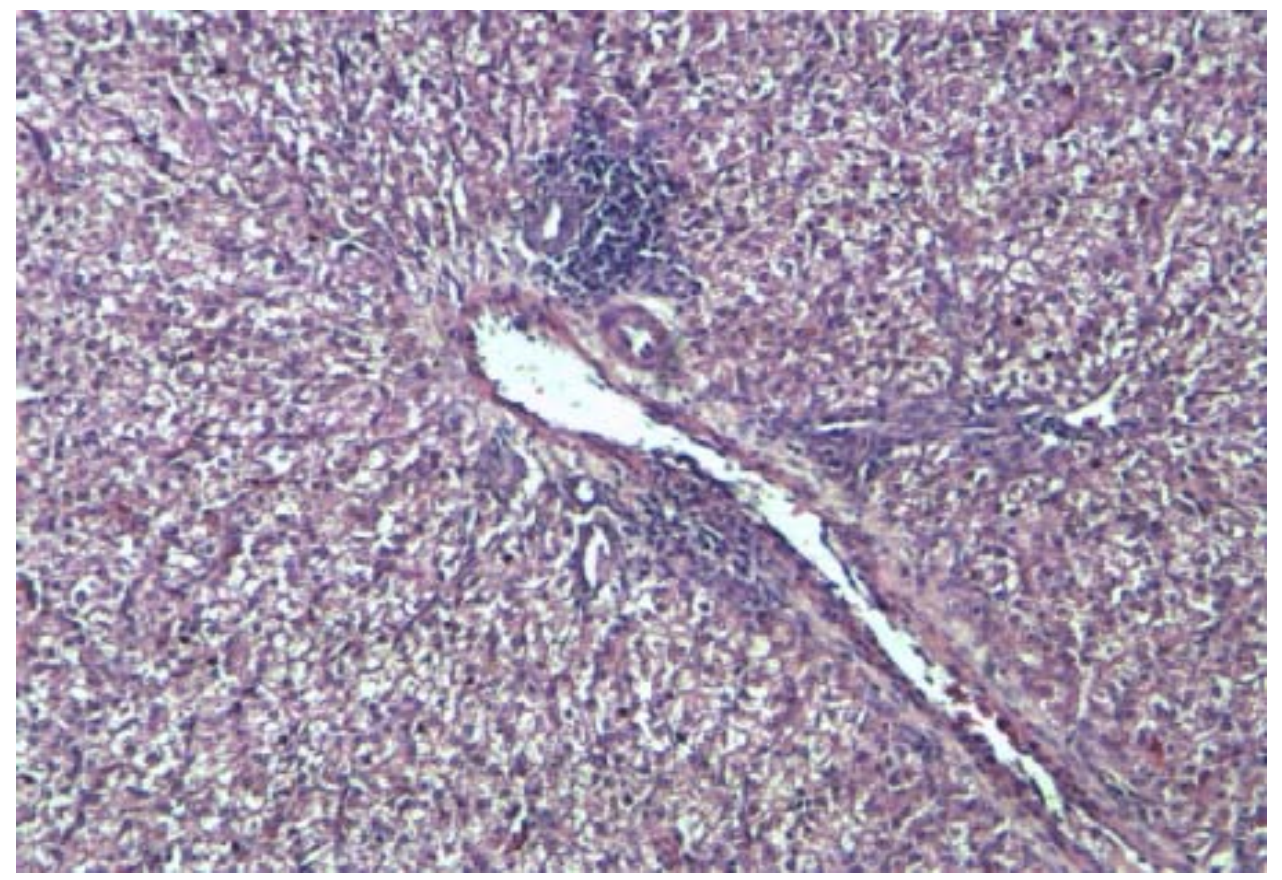

Figura 4. Histología de hígado de pollo de 14 días del grupo sin antibiótico promotor de crecimiento ni extracto de plantas. Presenta degeneración hidrópica y tumefacción turbia alrededor de triada hepática con presencia de nódulos linfocitarios (x100) 
et al. (2005) con aceite esencial de orégano al 0.1 y $0.075 \%$. Asimismo, se indica que el efecto de aceite esencial de orégano se puede potencializar con la adición de aceites esenciales de otras plantas (Barreto et al., 2008); sin embargo, Hernández et al. (2004) lo mezcló con canela y pimienta sin llegar a encontrar diferencias estadísticas en términos de ganancia de peso y conversión alimenticia, en tanto que Oetting et al. (2006), al mezclarlo con tomillo y clavo en dietas de lechones de 28 días obtuvo menor peso y mayor conversión alimenticia con relación al antibiótico.

Ensayos en pollos broilers utilizando $2 \mathrm{y}$ $5 \%$ de jengibre deshidratado en las dietas obtuvieron ligeras ventajas en términos de peso vivo y consumo de alimento, así como en rendimiento de carcasa, pero sin llegar a obtenerse diferencias estadísticas (AlHomidan et al., 2005; Zhang et al., 2009).

Al-Homidan et al. (2005) encontraron alteraciones en pollos con dietas suplementadas con $6 \%$ de jengibre deshidratado, tales como leve enteritis catarral, vacuolación citoplasmática de hepatocitos centrolobulillares y acumulación de linfocitos en el tracto portal hepático. En el presente estudio, en el día 14, las aves de los dos grupos con extractos de plantas medicinales presentaron severa degeneración hidrópica en hígado aunque sin diferencia con el grupo control APC; asimismo, en el día 42 se observó una regresión en la intensidad de estas dichas alteraciones, y sin llegar a afectar la ganancia de peso o la conversión alimenticia. Se presume que las alteraciones encontradas a los 14 días fueron producto de un factor común para todos los grupos y no producto de una intoxicación por las plantas utilizadas.

El patrón histomorfométrico con una tendencia a una mayor longitud de vellosidades intestinales y menor profundidad de criptas de Lieberkuhn lo presentó el grupo AEO, aunque sin diferencias significativas con los demás grupos. Fukayama et al. (2005) obtuvieron una mayor longitud de vellosidades con un mayor peso y menor conversión ali- menticia al día 42 al usar aceite esencial de orégano. Se sabe que la mayor longitud de la vellosidad intestinal provee una mayor área de absorción a la vellosidad; asimismo, el ancho de la vellosidad se relaciona a una menor hiperplasia a nivel de células caliciformes y a nivel de enterocitos, mejorando el sistema de absorción en el epitelio de revestimiento, pasando el nutriente directamente hacia la lámina propia, zona en donde se encuentran los capilares (Awad et al., 2009). Estudios en lechones también muestran un mejor desempeño con este patrón, ya que un aumento de la profundidad de las criptas podría ser consecuencia de un aumento en la descamación en la superficie de la vellosidad para generar una mayor renovación celular en la zona apical, por lo tanto menor absorción y una mayor pérdida energética (Oetting et al., 2006).

El efecto del desempeño del grupo SPC de los pollos en el presente estudio puede estar relacionado a las óptimas condiciones ambientales, las buenas prácticas de bioseguridad, y el estricto control sanitario realizado en la granja experimental, llevando esto a una reducción del desafío inmunológico y una disminución del riesgo de entrada de patógenos. En este mismo sentido, los resultados de los grupos AEO y JD pueden haber estado influenciados por el bajo reto sanitario debido a las condiciones máximas de manejo y bioseguridad, como corresponde a una granja experimental.

\section{Conclusiones}

- El uso de aceite esencial de orégano a $0.1 \%$ y del jengibre deshidratado al $1 \%$ en las dietas presentaron una respuesta productiva en pollos Cobb 500 similar a la obtenida con la dieta suplementada con antibiótico y a la dieta sin ningún tipo de promotor de crecimiento.

- Se encontraron diversas alteraciones tanto a nivel de vellosidades como de hepatocitos en todos los grupos en el día 
14, y una regresión de las lesiones en el día 42, sin diferencias significativas entre grupos.

\section{LiTERATURA CITADA}

1. Akoachere TK, Ndip RN, Chenwi EB, Ndip L.M, Njock TE, Anong, DN. 2002. Antibacterial effect of Zingiber officinale and Garcinia kola on respiratory tract pathogens. East Afr Med J 74: 588-592.

2. AL-Homidan A. 2005. Efficacy of using different sources and levels of Allium cepa, Allium Sativum and Zingiber officinale on broiler chicks performance. Saudi J Biol Sci 12: 96-102.

3. Albado E, Saez G, Grabriel S. 2001. Composición química y actividad antibacteriana del aceite esencial del Origanum vulgare (orégano). Rev Med Hered 12: 16-19.

4. Arcila C, Loarca G, Lecona S, González E. 2004. El orégano: propiedades, composición y actividad biológica de sus componentes. ALAN 4(1). [Internet], [08 de mayo de 2010]. Disponible en http://www.alanrevista.org/ ediciones/2004/oregano_propiedades_composicion_activi-dad biologica.asp

5. Awad WA, Ghareeb K, Abdel-Raheem S, Bohm J. 2009. Effects of dietary inclusion of probiotic and synbiotic on growth performance, organ weights, and intestinal histomorphology of broiler chickens. Poultry Sci 88: 49-55.

6. Barreto MSR, Menten JFM, Racanicci AMC, Pereira PWZ, Rizzo $P V .2008$. Plant extracts used as growth promoters in broilers. Braz J Poul Sci 10: 109-115.

7. Chen JC, Huang LJ, Wu SL, Kuo SC, Ho TY, Hsiang CY. 2007. Ginger and its bioactive component inhibit enterotoxigenic Escherichia coli heatlabile enterotoxin-induced diarrhea in mice. J Agric Food Chem 55: 8390-8397.
8. Dorman D, Deans S. 2000. Antimicrobial agents from plants: antibacterial activity of plant volatile oils. J Appl Microbiol 88: 308-316.

9. [FAO-OMS] Food and Agriculture Organization y Organización Mundial de la Salud. 2005. La necesidad de fortalecer los programas nacionales de monitoreo del uso de los antimicrobianos en medicina veterinaria en la Región. Conferencia Regional FAO/OMS sobre Inocuidad de los Alimentos para las Américas y el Caribe. San José de Costa Rica: FAO-OMS. 9 p.

10. Fukayama EH, Bertechini AG, Geraldo A, Kanji R, Murgas LDS. 2005. Extrato de orégano como aditivo em rações para frangos de corte. Rev Bras Zootec 34: 2316-2326.

11. Hashemi SR, Davoodi. 2010. Phytogenics as new class of feed additive in poultry industry. J Anim Vet Adv 9: 2295-2304.

12. Hernández F, Madrid J, García V, Orengo J, Megías MD. 2004. Influence of two plants extracts on broilers performance, digestibility and digestive organ size. Poultry Sci 83: 169-174.

13. Indu MN, Hatha AAM, Abirosh C, Harsha U, Vivekanandan G. 2006. Antimicrobial activity of some of the South-Indian spices against serotypes of Escherichia coli, Salmonella, Listeria monocytogenes and Aeromonas hydrophila. Braz J Microbiol 37: 153-158.

14. Lambert RJW, Skandamis PN, Coote PJ, Nychas G. 2001. A study of the minimum inhibitory concentration and mode of action of oregano essential oil, thymol and carvacrol. J Appl Microbiol 91: 453-462.

15. Morais J, Flores D, Pozzatti P, Moraes C, Rogérico P, Hartz S. 2007. Atividade antimicrobiana dos óleos essenciais de orégano, tomilho e canela frente a sorovares de Salmonella enterica de origem avícola. Ciência Rural 37: 803-808.

16. Moorthy M, Ravi S, Ravikumar M, Viswanathan K, Edwin S. 2009. Ginger, pepper and curry leaf powder as feed 
additives in broiler diet. Int J Poul Sci 8: 779-782.

17. Oetting LL, Utiyama CE, Giani PA, Ruiz U, Miyada VS. 2006. Efeitos de extratos vegetais e antimicrobianos sobre a digestibilidade aparente, o desempenho, a morfometria dos órgãos e a histologia intestinal de leitões recémdesmamados. Rev Bras Zootec 35: 1389-1397.

18. Ortiz PA. 2004. Utilización de alternativas naturales a los antibióticos promotores del crecimiento en la salud intestinal y parámetros productivos de pollos broiler. Tesis de Ingeniero Agrónomo. Valparaíso de Chile: Pontificia Universidad Católica de Valparaíso. 107 p.

19. Silva MA, Pessotti BM, Zanini SF, Colnago GL, Rodrigues MR, Nunes L, Zanini MS, Martins IV. 2009. Intestinal mucosa estructure of broiler chickens infected experimentally with Eimeria tenella and treated with essential oil of oregano. Ciência Rural 39: 1471-1477.

20. Stoyanova A, Denkova Z, Nenoy $N$, Slavchev A, Jirovetz L, Buchbauer G,
Lien HN, et al. 2006. C2H2F4-SCFEoleoresins of black pepper (Piper nigrum l.) and ginger (Zingiber officinale [1]) Rosc.) from Vietnam: Antimicrobial testing, gas chromatographic analysis and olfactoric evaluation. Electron J Environ Agric Food Chem 5: 1615-1623.

21. Torres C, Zarazaga M. 2002. Antibióticos como promotores del crecimiento en animales. ¿Vamos por el buen camino? Gac Sanit 16(2): 109-112.

22. [WHO] World Health Organization. 2001. Global strategy for containment of antimicrobial resistance. Switzerland: WHO. 99 p.

23. Windisch W, Schedler K, Plitzner C, Kroismayr. 2008. Use of phytogenic as feed additives for swine and poultry. $\mathrm{J}$ Anim Sci 86: 140-148.

24. Zhang G, Yang Z, Wang Y, Yang W, Jiang S, Gai G. 2009. Effects of ginger root (Zingiber officinale) processed to different particle sizes on growth performance, antioxidant status, and serum metabolites of broiler chickens. Poultry Sci 88: 2159-2166. 